\title{
IDEALS IN A POLYADIC ALGEBRA ${ }^{1}$
}

\section{FRED B. WRIGHT}

In an important sequence of papers, Halmos has developed the beautiful and significant theory of algebraic logic. The central mathematical object of this theory is the polyadic algebra, which incorporates in an axiomatic way the concepts of substitution, transformation, and quantification for the lower functional calculus. The beauty of the theory is revealed by the fact that the semantic completeness theorem of Gödel becomes the algebraic theorem that every polyadic algebra is semisimple.

The proof of semisimplicity is quite easy. The problem is reduced to proving that a monadic algebra is semisimple, and this in turn is reduced to Stone's theorem that any Boolean algebra is semisimple. The purpose of this note is to observe that this proof can be made to yield more information concerning the polyadic ideals of a polyadic algebra.

Lemma. Let $(A, I, S, \exists)$ be a polyadic algebra. Then a subset $J$ of the Boolean algebra $A$ is a polyadic ideal of $(A, I, S, \exists)$ if and only if $J$ is a monadic ideal of the monadic algebra $(A, \exists(I))$.

This is precisely the statement of [3, Lemma 8.1]. This comment, together with the definition of a polyadic algebra, is essentially all that is required from [3]. The reader familiar with the content of [1] will be able to follow the proofs below.

Let $(A, \exists)$ be a monadic algebra, and let $B$ be the set of all closed elements of $A$. That is, $p \in B$ if and only if $p=\exists p$. Then $B$ is the range of the quantifier $\exists$, and is a Boolean subalgebra of $A$. Let $J$ be a monadic ideal of $(A, \exists)$. This means that $J$ is an ideal of $A$ such that $p \in J$ implies that $\exists p \in J$. Clearly $J \cap B$ is an ideal of the Boolean algebra $B$. It is much more significant that we can pass from ideals of $B$ to monadic ideals of $(A, \exists)$.

Let $K$ be an ideal of $B$. We define a subset $K^{\exists}$ of $A$ by setting $K^{\exists}=\{p \in A: \exists p \in K\}$. Since $\exists \exists p=\exists p$, it follows that $K \subset K \exists$. Hence if $p \in K^{\exists}$, then $\exists p \in K^{\exists}$. If $p, q \in K^{\exists}$, then $\exists(p \vee q)=\exists p \vee \exists q$ is in $K$, so that $p \vee q \in K^{\exists}$. If $p \in K^{\exists}$ and if $q \leqq p$, then $\exists q \leqq \exists p$, so that $\exists q \in K$. Hence $q \in K^{\exists}$, and therefore $K^{\exists}$ is a monadic ideal of

Presented to the Society, December 1, 1956; received by the editors August 11, 1956.

1 This work was partially supported by the National Science Foundation grant to Tulane University. 
( $A, \exists$ ), with $K \subset K^{\exists}$. It follows that $K \subset K^{\exists} \cap B$. On the other hand, if $p \in B$, then $\exists p=p$, and if $p \in K \exists$, then $\exists p \in K$. Thus if $p \in K \exists \cap B$, then $\exists p=p \in K$. Hence $K \exists \cap B=K$.

If $J$ is a monadic ideal of $(A, \exists)$, it is quite easy to see that $(J \cap B) \exists$ $=J$. Clearly, in fact, we have $J \subset(J \cap B) \exists$. If, conversely, $p$ is in $(J \cap B) \exists$, then $\exists p \in J \cap B$, and in particular we have $\exists p \in J$. Then, since $p \leqq \nexists p$ and since $J$ is an ideal of $A$, it follows that $p \in J$. These remarks yield the following result.

TheOREm 1. Let $(A, \exists)$ be a monadic algebra, and let $B$ be the range of the quantifier $\exists$. Then the correspondences $J \rightarrow J \cap B$ and $K \rightarrow K^{\exists}$ are reciprocal one-to-one correspondences between the set of all monadic ideals $J$ of $(A, \exists)$ and the set of all ideals $K$ of $B$. These correspondences preserve inclusion and maximality.

REMARK. The above argument has been adapted from the proof of the semisimplicity of a monadic algebra given by Halmos [1, Theorem 7].

THEOREM 2. There exists an inclusion-preserving one-to-one correspondence between the polyadic ideals of a polyadic algebra $(A, I, S, \exists)$ and the ideals of the Boolean algebra $B$ of closed elements of $A$.

This is a corollary of Theorem 1 and the lemma. If $K$ is an ideal of $B$, the corresponding polyadic ideal may be described as the set of all $p \in A$ such that $\exists(I) p \in K$, or, alternatively, as the set of all $p \in A$ such that $\exists\left(I_{0}\right) p \in K$ for each subset $I_{0}$ of $I$. We may denote this polyadic ideal by $K^{\exists}$ without any danger of ambiguity.

Again, let $(A, I, S, \exists)$ be a polyadic algebra, and suppose that $M$ is an ideal of the Boolean algebra $A$. If $B$ denotes the subalgebra of closed elements, then $(M \cap B) \exists$ is a polyadic ideal of $(A, I, S, \exists)$. If $p \in(M \cap B) \exists$, then $\exists(I) p \in M \cap B$. Since $p \leqq \exists(I) p$ it follows that $p \in M$, and thus $(M \cap B){ }^{\exists} \subset M$. The inclusion-preserving nature of the above correspondence implies that the polyadic ideal $(M \cap B) \exists$ contains every other polyadic ideal which is contained in $M$. It is clear that if $M$ is a maximal ideal of $A$, then $(M \cap B){ }^{\exists}$ is a maximal polyadic ideal of $(A, I, S, \exists)$. Thus we have

Theorem 3. If $(A, I, S, \exists)$ is a polyadic algebra, then every ideal $M$ of $A$ contains a polyadic ideal $(M \cap B) \exists$ of $(A, I, S, \exists)$ which contains every other polyadic ideal contained in $M$; if $M$ is maximal, then $(M \cap B){ }^{\exists}$ is maximal.

The semisimplicity of a polyadic algebra is now a corollary. Since every ideal in a Boolean algebra is the intersection of all the maximal 
ideals which contain it, it follows that the intersection of all the maximal polyadic ideals is equal to the intersection of all the maximal ideals which contain a maximal polyadic ideal. By Theorem 3, this includes all maximal ideals, and by Stone's theorem, this intersection is the zero ideal.

The equivalence of simple algebras and simple quantifiers is also a detail of this approach. A quantifier is said to be simple if its range is a simple subalgebra of the given algebra. By Theorem 2, a polyadic algebra is simple if and only if the quantifier $\exists(I)$ is simple.

It may be worth noting that the functional representation of simple monadic algebras [1, Theorem 6] has a purely algebraic form which serves to reduce the representation problem to the Stone representation theorem for Boolean algebras.

Scholium. Two simple monadic algebras are monadically isomorphic if and only if they are isomorphic as Boolean algebras.

This does not hold for polyadic algebras, however.

\section{REFERENCES}

1. P. R. Halmos, Algebraic logic I. Monadic Boolean algebras, Compositio Math. vol. 12 (1955) pp. 217-249.

2. - The basic concepts of algebraic logic, Amer. Math. Monthly vol. 63 (1956) pp. 363-387.

3. - Algebraic logic II. Homogeneous locally finite polyadic Boolean algebras of infinite degree, Fund. Math. vol. 43 (1957) pp. 255-325.

Tulane University of Louisiana 